\title{
Extracting the parton distribution functions evolution equations using the stochastic modeling in the non-equilibrium statistical mechanics
}

\author{
N. Olanj $\mathrm{f}^{\dagger}$ E. Moradi ${ }^{\dagger}$, and M. Modarres ${ }^{\ddagger}$ \\ ${ }^{\dagger}$ Physics Department, Faculty of Science, \\ Bu-Ali Sina University, 65178, Hamedan, Iran and \\ ¥Physics Department, University of Tehran, 1439955961, Tehran, Iran.
}

\begin{abstract}
In this paper, using the stochastic modeling of the non-equilibrium statistical mechanics in the momentum space, the evolution equations of the parton distribution functions $(P D F)$ usually used in the hadrons phenomenology are generated. These stochastic modeling $P D F$ evolution equations are the same as those of the Dokshitzer-Gribov-Lipatov-Altarelli-Parisi (DGLAP) ones, but they can be obtained by a more simplistic mathematical procedure based on the non-equilibrium statistical mechanics and the theory of Markov processes.

PACS numbers: 87.10.Mn, 02.50.Ga, 12.38.Aw
\end{abstract}

Keywords: parton distribution function, $D G L A P$, non-equilibrium statistical mechanics, master equation, QCD, Markov processes, stochastic modeling.

${ }^{*}$ Corresponding author, Email : n_olanj@basu.ac.ir , Tel:+98-81-38381601 


\section{INTRODUCTION}

The integrated parton distribution functions $(P D F)$ are the main objects of phenomenological computations in the high energy collisions of particle physics. These PDF are usually obtained by using the experimental data via the parameterizations procedures which are constrained by the sum rules and a few theoretical assumptions, e.g., see the reference [1].

Today, many of the high-energy particle physics laboratories, including the $L H C$, use the PDF to describe and analyze their extracted data from the deep inelastic scatterings, e.g., in the various $L H C$ detectors such as ATLAS, CMS, LHCb and ALICE [2 5].

Because of the importance of this subject, the Dokshitzer-Gribov-Lipatov-Altarelli-Parisi (DGLAP) obtained the evolution equations for the $P D F$ in terms of $Q^{2}$ (the hard scale of probe) in the lowest order level of the quantum chromodynamics $(Q C D)$. The $D G L A P$ evolution equations are generated [6] by performing the Mellin transform techniques starting from the renormalization group equations, as follows:

$$
\begin{aligned}
& \frac{\partial q\left(x, Q^{2}\right)}{\partial \operatorname{Ln} Q^{2}}=\frac{\alpha_{s}\left(Q^{2}\right)}{2 \pi} \int_{x}^{1} \frac{d z}{z}\left[P_{q q}(z) q\left(\frac{x}{z}, Q^{2}\right)+P_{q g}(z) g\left(\frac{x}{z}, Q^{2}\right)\right], \\
& \frac{\partial g\left(x, Q^{2}\right)}{\partial \operatorname{Ln} Q^{2}}=\frac{\alpha_{s}\left(Q^{2}\right)}{2 \pi} \int_{x}^{1} \frac{d z}{z}\left[P_{g g}(z) g\left(\frac{x}{z}, Q^{2}\right)+P_{g q}(z) \sum_{q} q\left(\frac{x}{z}, Q^{2}\right)\right],
\end{aligned}
$$

where $q\left(x, Q^{2}\right)$ and $g\left(x, Q^{2}\right)$ are the quark or anti-quark and the gluon distribution functions, respectively. $P_{a a^{\prime}}(z)\left(a, a^{\prime}=q, \bar{q}, g\right)$, the probability of emitting parton $a$ with fraction $z$ of the longitudinal momentum of the parton $a^{\prime}$, are the leading order $(L O)$ splitting functions. $Q^{2}$ is the hard scale of the scattering that comes from the virtuality of the space like exchanged photon $\left(q^{2} \equiv q^{\mu} q_{\mu}=-Q^{2}\right)$, and $x$ is a fraction of the longitudinal momentum

of the parent hadron (the Bjorken variable). In the other words, $q_{i}\left(x, Q^{2}\right)\left(g\left(x, Q^{2}\right)\right)$ is the probability of the finding the quark or anti-quark of type $i$ (gluon) with the fraction $x$ of longitudinal momentum of the parent hadron inside the hadron by probing photon with the energy scale $Q^{2}$. It is worth noting that Kimber, Martin and Ryskin $(K M R)$ [10, 11] modified the standard DGLAP equations due to the separation of virtual and real parts of the evolutions. Therefore, the $D G L A P$ equation is rewritten as follows:

$$
\frac{\partial a\left(x, Q^{2}\right)}{\partial \operatorname{Ln} Q^{2}}=\frac{\alpha_{s}\left(Q^{2}\right)}{2 \pi} \sum_{a^{\prime}=q, g}\left[\int_{0}^{1-\Delta} d z P_{a a^{\prime}}(z) a^{\prime}\left(\frac{x}{z}, Q^{2}\right)-a\left(x, Q^{2}\right) \int_{0}^{1-\Delta} d z P_{a^{\prime} a}(z)\right]
$$


where $a\left(x, Q^{2}\right)$ denotes $x g\left(x, Q^{2}\right)$ or $x q\left(x, Q^{2}\right)$ and $\Delta$ is a cut-off to prevent $z=1$ singularities in the splitting functions which arises from the soft gluon emissions.

Also, the $Q^{2}$ evolution of the $P D F$ determined by $Q C D$ were used to investigate the deep inelastic structure function $\left(F_{2}\left(x, Q^{2}\right)\right)$ of the proton [12]. The resulting evolution equation in the leading order level is the same as the DGLAP evolution equations. In the present phenomenological studies, it is observed that the $P D F$ with the increasing the hard scale $Q^{2}$ of the probe never reaches a stable state. So one can conclude that the prominent role of non-equilibrium statistical mechanics in describing the evolution of the $P D F$ in the hadron is vital. Then it would be interesting to show that the above evolution equation can be obtained, using some simplistic stochastic modeling based on the non-equilibrium statistical mechanics.

Therefore, in the reference [13], Nayak studied the splitting functions in the non-equilibrium $Q C D$ at the leading order level and Buccella et al, in the reference [14, obtained the low $Q^{2}$ boundary conditions for DGLAP equations by using the quantum statistical mechanics. So, considering that, the process of the parton $Q^{2}$ evolution in the leading order level has completely stochastic nature, and depends only on a previous step, i.e., Markov processes [15], in this work, we intend to study the $Q^{2}$ evolution of the $P D F$ in the non-equilibrium $Q C D$ by using the stochastic modeling. It is shown that, these evolution equations are the same as those of DGLAP, but they can be generated by a much "simpler" mathematical procedure, using the stochastic modeling of the non-equilibrium statistical mechanics and the theory of Markov processes.

The parton $Q^{2}$ evolution equations derived from this procedure are expressed by using the master equations (the evolution equation of stochastic variables of Markov processes) of statistical mechanics of systems far from equilibrium in the momentum space. This master equation is equivalent to the master equation in the position space for the singleparticle systems with the multi-species reaction diffusion processes on the one-dimensional finite continuous lattice. It should be noted that the master equation in the position space (momentum space) is an integro-differential equation governing the time (energy) evolution of the probability [16].

So the paper is organized as follows: the Sect. $I I$ contains an overview of the master equation of Markov processes. Sect. III is devoted to study the $Q^{2}$ evolution of the PDF inside the hadron by using stochastic modeling in the non-equilibrium statistical mechanics and the 
theory of Markov processes. Finally, Sect. IV contains results, discussions and conclusions.

\section{AN OVERVIEW OF DERIVATION OF THE MASTER EQUATION OF MARKOV PROCESSES}

The master equation is the evolution equation of stochastic variables of Markov processes. It should be noted that Markov processes are stochastic processes that depend only on a limited history of evolution. The master equation is one of the most important statistical physics equations due to its many applications in various sciences areas. In this section, we take a brief look at how to derive the master equation of non-equilibrium statistical mechanics in the position space [16].

First, we define the notations for the probability density, the joint probability density and the conditional probability density for the stochastic variable $Y$, respectively, as follows:

(i) $P_{1}\left(y_{1}, t_{1}\right)$ : the probability density that $Y$ has a value $y_{1}$ at time $t_{1}$,

(ii) $P_{2}\left(y_{1}, t_{1} ; y_{2}, t_{2}\right)$ : the joint probability density that $Y$ has a value $y_{1}$ at time $t_{1}$ and a value $y_{2}$ at time $t_{2}$,

(iii) $P_{1 \mid 1}\left(y_{1}, t_{1} \mid y_{2}, t_{2}\right)$ : the conditional probability density that $Y$ has a value $y_{2}$ at time $t_{2}$ given that it had a value $y_{1}$ at time $t_{1}$, so that the probability density is normalized as:

$$
\int d y_{1} P_{1}\left(y_{1}, t_{1}\right)=1
$$

and the joint probability density can be reduced, as follows:

$$
\int d y_{2} P_{2}\left(y_{1}, t_{1} ; y_{2}, t_{2}\right)=P_{1}\left(y_{1}, t_{1}\right)
$$

also, the conditional probability density satisfies the following equation:

$$
P_{1}\left(y_{1}, t_{1}\right) P_{1 \mid 1}\left(y_{1}, t_{1} \mid y_{2}, t_{2}\right)=P_{2}\left(y_{1}, t_{1} ; y_{2}, t_{2}\right)
$$

Finally, by combining equations (4) and (5), we arrive at the following relation between probability densities at different times:

$$
P_{1}\left(y_{2}, t_{2}\right)=\int d y_{1} P_{1}\left(y_{1}, t_{1}\right) P_{1 \mid 1}\left(y_{1}, t_{1} \mid y_{2}, t_{2}\right) .
$$

It should be noted that equation (6) is obtained assuming that the stochastic variable $Y$ is continuous. If we consider the stochastic variable $Y$ to be discrete, the equation (6) simply 
becomes as following:

$$
P_{1}(n, t+\Delta t)=\sum_{m=1}^{M} P_{1}(m, t) P_{1 \mid 1}(m, t \mid n, t+\Delta t),
$$

where $M$ is the total number of states. Using the derivative definition, the differential equation for $P_{1}(n, t)$ can be obtained from the equation (7):

$$
\begin{aligned}
\frac{\partial P_{1}(n, t)}{\partial t} & =\lim _{\Delta t \rightarrow 0}\left(\frac{P_{1}(n, t+\Delta t)-P_{1}(n, t)}{\Delta t}\right) \\
& =\lim _{\Delta t \rightarrow 0} \frac{1}{\Delta t} \sum_{m=1}^{M} P_{1}(m, t)\left(P_{1 \mid 1}(m, t \mid n, t+\Delta t)-\delta_{m n}\right) .
\end{aligned}
$$

By expanding the transition possibility $P_{1 \mid 1}(m, t \mid n, t+\Delta t)$ in a power series in $\Delta t$ and keeping only the lowest order term, the transition possibility becomes:

$$
P_{1 \mid 1}(m, t \mid n, t+\Delta t)=\delta_{m n}\left[1-\Delta t \sum_{\ell=1}^{M} \omega_{m, \ell}(t)\right]+\omega_{m, n}(t) \Delta t,
$$

where $\omega_{m, n}(t)$ is the transition probability rate. Therefore, the first term on the right of the equation (9) $\left(\delta_{m n}\left[1-\Delta t \sum_{\ell=1}^{M} \omega_{m, \ell}(t)\right]\right)$ is the probability that no transition occurs at time interval $t$ to $t+\Delta t$, and the second term $\left(\omega_{m, n}(t) \Delta t\right)$ implies the probability of a transition from state $m$ to state $n$ at time interval $t$ to $t+\Delta t$.

By combining the equation (8) and the equation (9), and given that we have only one transition (Markov processes) in the equation (9), we obtain the master equation of Markov processes for the discrete stochastic variable, as follows:

$$
\frac{\partial P_{1}(n, t)}{\partial t}=\sum_{m=1}^{M}\left[P_{1}(m, t) \omega_{m, n}(t)-P_{1}(n, t) \omega_{n, m}(t)\right]
$$

The master equation (the equation (10) ) describe the time evolution of the probability $P_{1}(n, t)$ due to transitions into the state $n$ from all others states (the first $M$ terms on the right which are called source terms) and due to transitions out of state $n$ into all others states (the second $M$ terms on the right which are called sink terms). Assuming the stochastic variable is continuous, the equation 10 for the infinite continuous stochastic variable $X$ is written as follows:

$$
\frac{\partial P_{1}(x, t)}{\partial t}=\int_{-\infty}^{+\infty} d x^{\prime}\left[P_{1}\left(x^{\prime}, t\right) \omega\left(x^{\prime} \mid x, t\right)-P_{1}(x, t) \omega\left(x \mid x^{\prime}, t\right)\right],
$$


where $\omega\left(x^{\prime} \mid x, t\right)$ is the transition rate. Now considering that the value of the stochastic variable $X$ changes from $x$ to $x^{\prime}=x+y$ at a transition and by introducing the notation $\tau(x, y, t)=\omega(x \mid x+y, t)$, the master equation can be rewritten as follows:

$$
\frac{\partial P_{1}(x, t)}{\partial t}=\int_{-\infty}^{+\infty} d y\left(P_{1}(x-y, t) \tau(x-y, y, t)-P_{1}(x, t) \tau(x, y, t)\right) .
$$

Finally, the generalization of the master equation (12) in the position space, i.e., the stochastic variable $X$ represents the position of the particle, for the single-particle systems with the $p$-species reaction diffusion processes is as follows [16, 17]:

$$
\frac{\partial P_{i}(x, t)}{\partial t}=\sum_{j=1}^{p}\left[\int_{-\infty}^{+\infty} d y\left(P_{j}(x-y, t) \tau_{i j}(x-y, y, t)-P_{i}(x, t) \tau_{j i}(x, y, t)\right)\right]
$$

where $P_{i}(x, t)$ is the probability of finding at time $t$ the particle of type $i$ at the point $x$ and $\tau_{j i}(x, y, t)$ is the process rate that changes the position of the particle from $x$ to $x+y$ and the particle type from $i$ to $j$ at the time $t$. It should be noted that the first $p$ terms are the sources of $P_{i}(x, t)$ and the second $p$ terms are the sinks of it.

\section{THE STUDY OF THE PARTON $Q^{2}$ EVOLUTION EQUATIONS IN THE NON- EQUILIBRIUM $Q C D$ BY USING STOCHASTIC MODELING}

Based on the master equation (equation (13)), probing the structure of the hadron, by a virtual photon with virtuality $Q^{2}$ that coming from the projectile, e.g., electron, in the deep inelastic scattering, can be viewed as a portion of the parton evolution chain demonstrated in the figure 1 [18]. The splitting process at each stage of the evolution is a completely stochastic process and in each splitting, the fraction of the longitudinal momentum decreases, so:

$$
x<x^{\prime}<x^{\prime \prime}<x^{\prime \prime \prime}<\ldots<\xi
$$

According to this diagram, a parton on the one-dimensional finite $(0<x<1)$ continuous lattice (longitudinal momentum axis) changes its longitudinal momentum and type (convert quark to gluon and vice versa- flavour of quark) during the reaction diffusion processes to the scale $Q^{2}$.

According to the experimental evidence, the probability of the finding a parton type $i$ with 


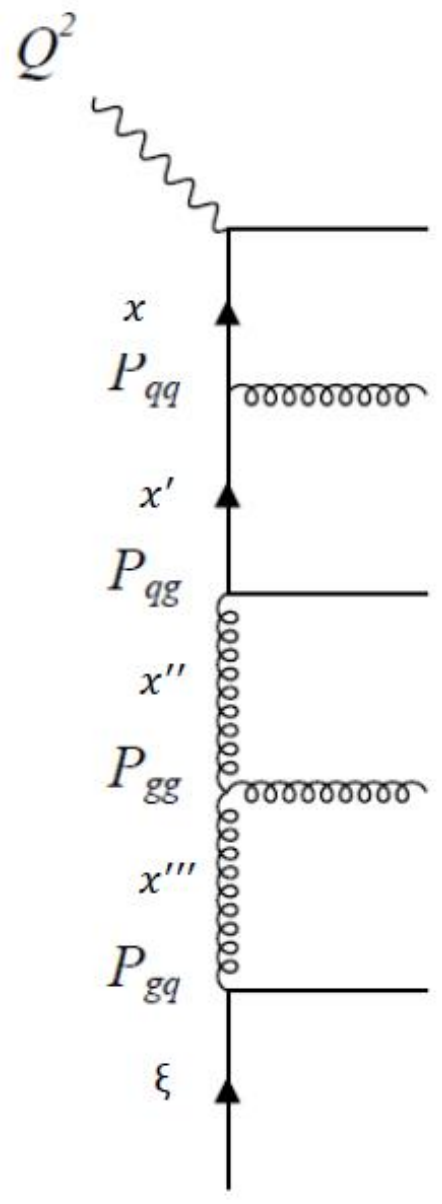

FIG. 1: A portion of the evolution chain (the different types of parton splitting in the evolution chain at the scale $Q^{2}[18]$ ).

the fraction $x$ of longitudinal momentum (the Bjorken variable) of the parent hadron inside the hadron with the increase in the energy of the virtual photon $\left(Q^{2}\right)$ never gets into a stable state [19]. In other words, the parton structure of the hadrons will never be saturated with increasing the energy of the virtual photon $\left(Q^{2}\right)$. Therefore, the process of the parton 
$Q^{2}$ evolution follows the non-equilibrium statistical mechanics and can satisfy the master equation (equation (13)) conditions.

Of course, it should be noted that, at the leading order level of the $Q C D$, the probability of finding a parton type $i$ that carrying the fraction $x$ of longitudinal momentum of the parent hadron in the hard scale $Q^{2}$ of the probe, depends only on a previous step and not on the history of evolution, i.e., Markov processes.

Then, the processes performed by the partons, corresponding to transitions between different positions in the master equation in the position space (13) with the transition rate $\tau_{j i}(x, y, t)$, at the leading order level inside the hadron can be expressed as follows:

$$
\begin{aligned}
& \text { 1) } g\left(\frac{x}{z}, Q^{2}\right) \longrightarrow q_{i^{\prime}}\left(x, Q^{2}\right) \text { with rate } \frac{\alpha_{s}\left(Q^{2}\right)}{2 \pi} P_{q g}(z) \text {, } \\
& \text { 2) } q_{i}\left(\frac{x}{z}, Q^{2}\right) \longrightarrow g\left(x, Q^{2}\right) \text { with rate } \frac{\alpha_{s}\left(Q^{2}\right)}{2 \pi} P_{g q}(z) \text {, } \\
& \text { 3) } q_{i}\left(\frac{x}{z}, Q^{2}\right) \longrightarrow q_{i}\left(x, Q^{2}\right) \text { with rate } \frac{\alpha_{s}\left(Q^{2}\right)}{2 \pi} P_{q q}(z) \text {, } \\
& \text { 4) } g\left(\frac{x}{z}, Q^{2}\right) \longrightarrow g\left(x, Q^{2}\right) \text { with rate } \frac{\alpha_{s}\left(Q^{2}\right)}{2 \pi} P_{g g}(z) \text {, }
\end{aligned}
$$

where $i^{\prime}$ change from 1 to $n_{F}$, that $n_{F}$ is the number of flavours of quark-antiquark pairs available to split the gluon into them and $i$ change from 1 to $2 n_{F}$ (the number of quarks and antiquarks of all flavours). It should be noted that in the cases 1 and 2 (the birth-death processes), in addition to the longitudinal momentum variation of the parton, the type of parton can be also changed, while the cases 3 and 4 are the diffusion processes in the momentum space, i.e., only the longitudinal motion changes. It should be noted that according to the figure 1, the birth-death processes are accompanied by the emission of a quark, and the diffusion processes are accompanied by the emission of a gluon, which are not considered in the parton evolution chain (Markov chain).

Since the rate of the reaction diffusion processes of the partons inside the hadron, $\frac{\alpha_{s}\left(Q^{2}\right)}{2 \pi} P_{a a^{\prime}}(z)$, is determined by using the phenomenological methods, the master equation for the $Q^{2}$ evolution of the $P D F$ is a phenomenological equation. As a result, the process of the parton $Q^{2}$ evolution in the leading order $(L O)$ level of the $Q C D$ is completely stochastic and depends only on the previous step. Therefore, it is the type of Markov processes in the statistical mechanics, and the master equation in the momentum space governs its $Q^{2}$ evolution. According to the $Q C D$, the strong force between partons becomes very weak and asymptotically toward zero at short distances inside the hadron $\left(d<<10^{-15} \mathrm{~m}\right)$. On 
the other hand, the high-energy experiments examine the structure of the hadron in a short time scales. Thus, the interactions between the partons can be ignored in comparison with their high energy interactions with the virtual photon [20]. In other words, with good approximation, the partons can be considered as the free particles inside the hadron i.e., the Feynman parton model [20]. Thus, the $Q^{2}$ evolution of partons can be studied in singleparticle systems.

Based on mentioned points, in the study of the PDF, we can model the parton $Q^{2}$ evolution in the leading order level of the $Q C D$ with the single-particle systems with the multi-species reaction diffusion processes, i.e., the birth-death processes (the cases 1 and 2 of equation (15) and the diffusion processes (the cases 3 and 4 of equation (15), on the finite onedimensional continuous lattice in the momentum space (the longitudinal momentum axis). The master equation $(13)$ in the momentum space for the finite continuous stochastic variable of the fractional longitudinal momentum $x$ (corresponding to the site $x$ in the position space) at the hard scale $\mu$ (corresponding to the time scale $t$ in the position space) is rewritten as follows:

$$
\frac{\partial P_{i}(x, \mu)}{\partial \mu}=\sum_{j=1}^{p}\left[\int_{0}^{1} d z\left(P_{j}\left(\frac{x}{z}, \mu\right) \tau_{i j}(z, \mu)-P_{i}(x, \mu) \tau_{j i}(z, \mu)\right)\right]
$$

where $P_{i}(x, \mu)$ is the probability of the finding the parton of type $i$ with the fraction $x$ of longitudinal momentum of the parent hadron inside the hadron by probing photon with the energy scale $\mu$. Also, $\tau_{i j}(z, \mu)$ is the rate of transition $P_{j}\left(\frac{x}{z}, \mu\right) \rightarrow P_{i}(x, \mu)$, by the emission of a parton that is not considered in the parton evolution chain. It should be noted that this transition occurs by emitting parton $i$ (with the fraction of longitudinal momentum $x$ ) with fraction $z$ of the longitudinal momentum of the parton $j$ (with the fraction of longitudinal momentum $\left.\frac{x}{z}\right)$. Finally, by replacing $x q_{i}(x, \mu)$ and $x g(x, \mu)$ instead of $P_{i}(x, \mu), \frac{\alpha_{s}(\mu)}{2 \pi} P_{i j}(z)$ instead of $\tau_{i j}(z, \mu)$ and choosing the scale $\mu$ as $\operatorname{Ln}\left(\frac{Q^{2}}{Q_{0}{ }^{2}}\right)$ in the master equation 16 , the equations for the $Q^{2}$ evolution of the $P D F$ by considering this fact that the strong force does not change the flavour of the quark (consequently, for example in the case of the quark distribution functions, $\sum_{j=1}^{p}$ in the master equation 16 is converted to $\sum_{j=1}^{2}$, where 
$P_{1}(x, \mu)=x q_{i}\left(x, Q^{2}\right)$ and $\left.P_{2}(x, \mu)=x g\left(x, Q^{2}\right)\right)$, are obtained as follows:

$$
\begin{aligned}
\frac{\partial q_{i}\left(x, Q^{2}\right)}{\partial \operatorname{Ln} Q^{2}} & =\frac{\alpha_{s}\left(Q^{2}\right)}{2 \pi} \int_{0}^{1-\Delta} \frac{d z}{z}\left[P_{q q}(z) q_{i}\left(\frac{x}{z}, Q^{2}\right)+P_{q g}(z) g\left(\frac{x}{z}, Q^{2}\right)\right] \\
& -\frac{\alpha_{s}\left(Q^{2}\right)}{2 \pi} \int_{0}^{1-\Delta} d z\left[P_{q q}(z) q_{i}\left(x, Q^{2}\right)+P_{g q}(z) q_{i}\left(x, Q^{2}\right)\right] \\
\frac{\partial g\left(x, Q^{2}\right)}{\partial \operatorname{Ln} Q^{2}} & =\frac{\alpha_{s}\left(Q^{2}\right)}{2 \pi} \int_{0}^{1-\Delta} \frac{d z}{z}\left[\sum_{i=1}^{2 n_{F}}\left(P_{g q}(z) q_{i}\left(\frac{x}{z}, Q^{2}\right)\right)+P_{g g}(z) g\left(\frac{x}{z}, Q^{2}\right)\right] \\
& -\frac{\alpha_{s}\left(Q^{2}\right)}{2 \pi} \int_{0}^{1-\Delta} d z\left[n_{F} P_{q g}(z) g\left(x, Q^{2}\right)+P_{g g}(z) g\left(x, Q^{2}\right)\right] .
\end{aligned}
$$

One then can recognize the positive terms as the sources of the PDF and the negative terms as the sinks of it. Since $z=1$ does not represent any reaction diffusion process, $\Delta$ is entered at the upper limit of the integrals. The evolution equations obtained from stochastic modeling (the equation (17)) are the same as the DGLAP evolution equations, but obtained much easier. It should be noted that according to the evolution equation (17), which is derived from (1) the non-equilibrium statistical mechanics, and (2) the results of reference [14] in relation to the boundary conditions governing this evolution equation, we hope that in the near future, by solving methods of the master equation (for example by the coordinate Bethe ansatz method, see the reference [17], and the recursive method, see the reference [21]), the new solutions for the PDF are presented.

\section{RESULTS, DISCUSSIONS AND CONCLUSIONS}

It is shown that the process of the parton $Q^{2}$ evolution in the leading order level of the $Q C D$ is completely stochastic and depends only on a previous step (Markov processes). The parton $Q^{2}$ evolution in the leading order level of the $Q C D$ occurs by the emission of a parton, which changes the type and longitudinal momentum of the parton by the emission

of a quark (the birth-death processes) or the longitudinal momentum of the parton by the emission of a gloun (the diffusion process). Therefore, the master equation in the momentum space governs its $Q^{2}$ evolution. It should be noted, based on the Feynman parton model [20], the partons can be considered as the free particles inside the hadron.

Therefore, the parton $Q^{2}$ evolution in the leading order level of the $Q C D$ can be modeled by the single-particle systems with the multi-species reaction diffusion processes on the finite 
one-dimensional continuous lattice (the longitudinal momentum axis) in the momentum space.

In conclusion, considering the outcome of the section $I I I$, we extracted the PDF evolution equations by using the stochastic modeling. These evolution equations are the same as the DGLAP evolution equations, but they are obtained by much simpler mathematical procedure based on the non-equilibrium statistical mechanics and the theory of Markov processes. We hope in the near future, by solving methods of the master equation, the new solutions for the PDF are presented.

\section{Acknowledgments}

NO would like to acknowledge the University of Bu-Ali Sina for their support. MM would also like to acknowledge the Research Council of the University of Tehran for the grants provided for him.

[1] L. A. Harland-Long et al, Eur. Phys. J. C 75 (2015) 204.

[2] G. Pancheri and Y. N. Srivastava, Eur. Phys. J. C 77 (2017) 150.

[3] M. Klasen,Rev. Mod. Phys. 74 (2002) 1221.

[4] M. Modarres et al J. Phys. G: Nucl.Part., 46 (2019) 105005.

[5] N. Olanj and M. Modarres, Eur. Phys. J. C 79 (2019) 615.

[6] V. N. Gribov and L. N. Lipatov, Yad. Fiz. 15 (1972) 781.

[7] L. N. Lipatov, Sov. J. Nucl. Phys. 20 (1975) 94.

[8] G. Altarelli and G. Parisi, Nucl. Phys. B 126 (1977) 298.

[9] Y. L. Dokshitzer, Sov. Phys. JETP 46 (1977) 641.

[10] M. A. Kimber, A. D. Martin, and M. G. Ryskin, Phys. Rev. D 63 (2001) 114027.

[11] A. D. Martin, M. G. Ryskin, and G.Watt, Eur. Phys. J. C 66 (2010) 163.

[12] F. Halzen and A. D. Martin, "Quarks and Leptons: An Introductory Course in Modern Particle Physics," Wiley (1984).

[13] G. C. Nayak, Phys. Part. Nucl. 43 (2012) 742.

[14] F. Buccella, S. Sohaily, and F. Tramontano, Journal of Statistical Mechanics: Theory and 
Experiment (2019), https://doi.org/10.1088/1742-5468/ab054e.

[15] E. B. Dynkin, "Theory of Markov Processes", Dover publications (2006).

[16] L. E.Reichl, "A Modern Course in Statistical Physics", Wiley (2009).

[17] M. Alimohammadi and N. Ahmadi, J. Phys. A: Math. Gen. 35 (2002) 1325.

[18] M.A. Kimber, "Unintegrated Parton Distributions," Ph.D. Thesis, University of Durham (2001).

[19] J. C. Collins, "Foundation of Perturbative QCD", Cambridge University Press (2011).

[20] R. P. Feynman, "Photon-Hadron Interaction", Benjamin, New York (1972).

[21] M. Alimohammadi and N. Olanj, Physica A 389 (2010) 1549. 